\title{
Pertrochantäre Femurfraktur
}

\author{
Dankward Höntzsch
}

Im Februar 2005: per- und subtrochantäre Fraktur rechts bei einer zum Unfallzeitpunkt 70-jährigen Patientin, AOKlassifikation 31 A23 oder A33 (Abb.1).

Erstversorgung mit proximalem Femurnagel mit zusätzlich 2 Cerclagen, welche nur marginal helfen, die Fraktur zusätzlich zu stabilisieren. Die Fraktur konnte nicht anatomisch gestellt werden, es verbleibt ein ungünstiger leichter Varus und eine an sich günstige leichte $\mathrm{Me}$ dialisierung des Schaftes (Abb.2). Die knöcherne Heilung war verzögert. Die Funktion war ohne Belastung schmerzarm und weitgehend frei. Die Patientin ist nicht zur Vollbelastung gekommen.

Im 6. Monat, als bereits eine Osteosynthese und/oder Spongiosaplastik diskutiert wurde, kam es zum Implantatversagen des proximalen Femurnagels (Abb. 3).

Es wurde eine Reosteosynthese mit zunächst schwieriger Metallentfernung, Dekortikation, Valgisation und Osteosynthese mit gespannter langer Kondylenplatte durchgeführt (Abb. 4).

Bereits 8 Wochen später kam es bei mutig erlaubter zunehmender Belastung zum Plattenbruch in Höhe der Pseudarthrose (Abb. 5).

Es wurde eine Reosteosynthese mit deutlicher Valgisation, autologer Spongiosaplastik und gespannter aufgebogener Kondylenplatte durchgeführt (Abb. 6).

Zehn Monate nach Unfall war bei radiologisch vermeintlich ausreichendem Durchbau die Vollbelastung schmerzarm möglich (Abb. 7).

OP-JOURNAL 2008; 24: 220-221

(c) Georg Thieme Verlag KG Stuttgart • New York DOI 10.1055/s-2008-1039092

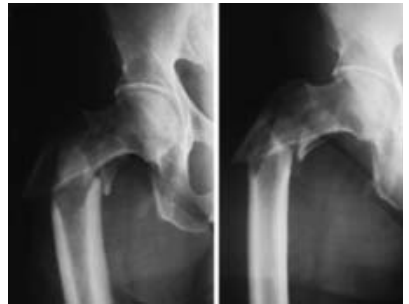

Abb. 1 Per/subtrochantäre Femurfraktur rechts.

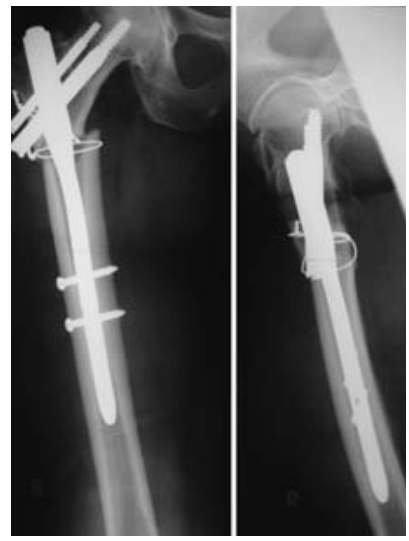

Abb. 2 Erstversorgung mit PFN und Cerclagen.

Vier Wochen später, im 11. Monat nach Unfall und Erstversorgung, kam es erneut zum Implantatversagen (Abb. 8).

Der Allgemeinzustand, die Compliance der Patientin sowie die lokalen Weichteilverhältnisse haben eine erneute Reosteosynthese erlaubt: Erneute Dekortikation, autologe Spongiosaplastik, zusätzliche Valgisation und Osteosynthese mit aufgebogener gespannter Kondylenplatte und zusätzlich Zuggurtung (Abb.9).

Bei Teilbelastung mit halbem Körpergewicht kam es 8 Wochen später, inzwischen 13 Monate nach Unfall, wiederum zum Implantatversagen (Abb. 10).
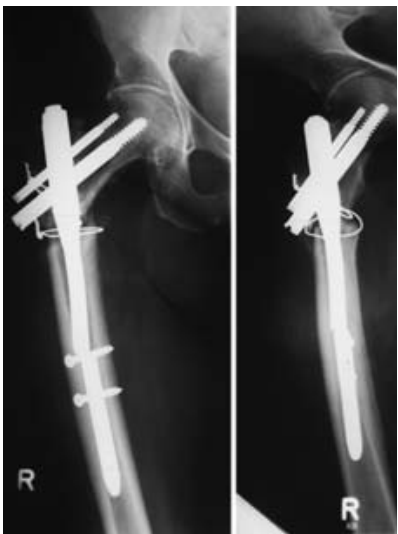

Abb. 3 Erstes Versagen nach 6 Monaten.

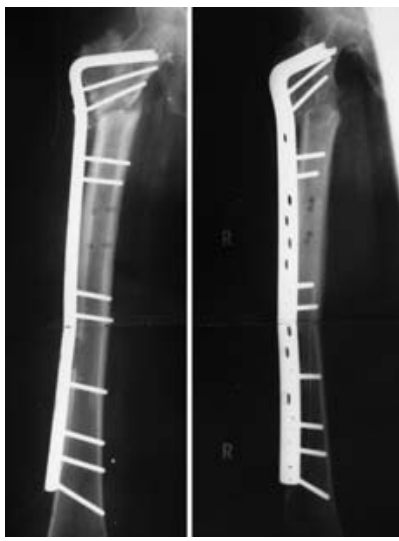

Abb. 4 Erste Reosteosynthese.

In Abwägung der möglichen Lösungen entschied man sich für eine erneute Reosteosynthese mit Dekortikation, autologer Spongiosaplastik, nochmaliger Valgisation und Medialisierung des Schaftes (Abb.11).

Der Heilungsverlauf der Wund- und Weichteile gestaltete sich erfreulicherweise wiederum problemlos. Es ließ sich rückwirkend betrachtet das erste Mal eine zügige knöcherne Überbrückung im Osteotomiebereich und periostal erkennen. Dies ging einher mit einer schmerzarmen zunehmenden, auch sub- 


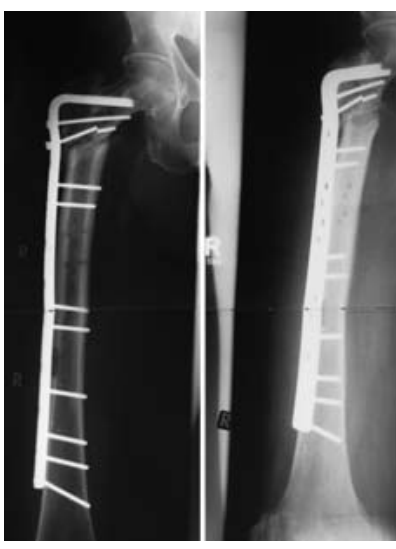

Abb. 5 Zweites Versagen nach 8 Wochen.

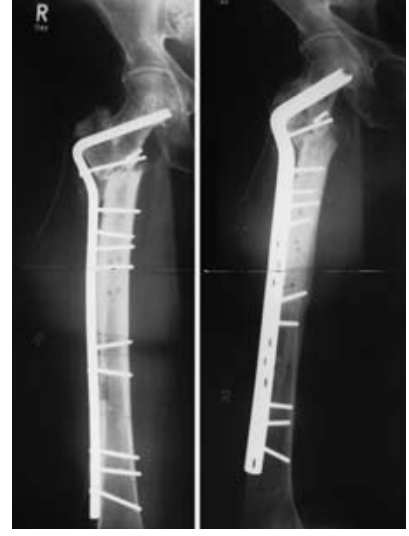

Abb. 6 Zweite Reosteosynthese.

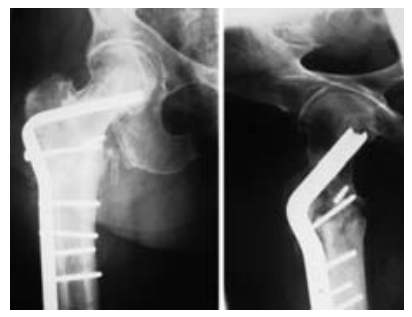

Abb. 7 Zehn Monate nach Unfall Vollbelastung.

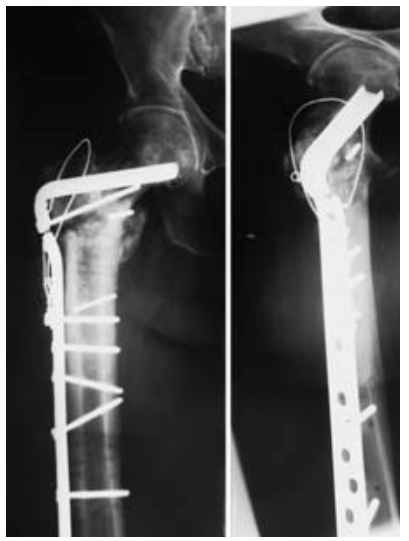

Abb. 10 Viertes Implantatversagen.

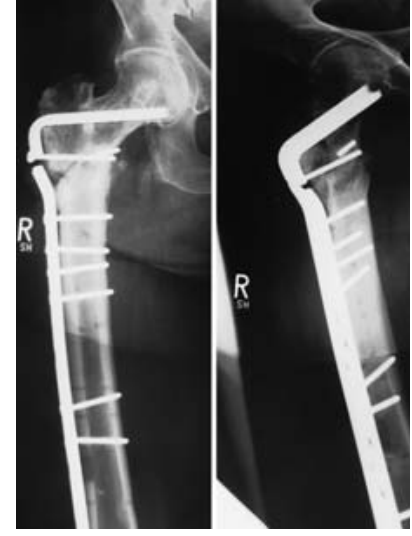

Abb. 8 Drittes Implantatversagen.

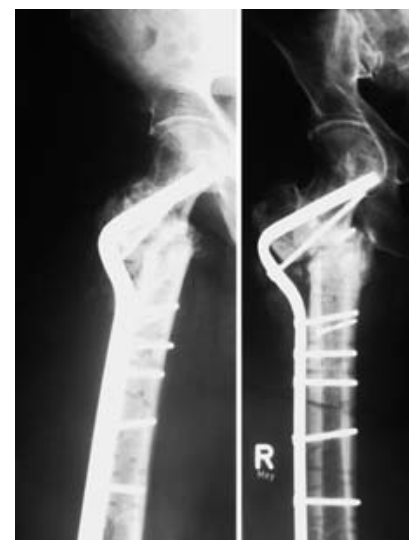

Abb. 11 Vierte Reosteosynthese mit erneuter Valgisation.

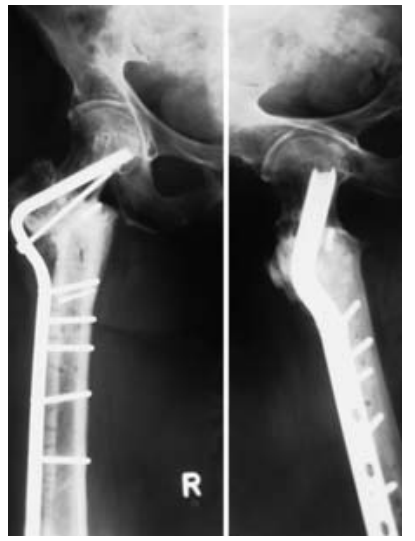

Abb. 12 Zwanzig Monate nach Unfall und 4 Reosteosynthesen endllich stabile Ausheilung.

jektiv von der Patientin verspürten Belastungsfähigkeit.

Zwanzig Monate nach dem Unfall war die Belastung schmerzarm möglich, die Fraktur war radiologisch sicher und endgültig durchbaut (Abb.12).
Die Funktion war für die Ab- und Anspreizung sowie die Rotation hälftig eingeschränkt. Die Streckung war frei, die Beugung mäßig eingeschränkt. Der Einsatz im tagtäglichen Leben war schmerzarm bis schmerzfrei möglich.

Fazit

- Es handelt sich um eine Frakturform, mit welcher wir uns täglich auseinandersetzen müssen.

- Die Versorgung mit proximalem Femurnagel ist eine Methode der ersten Wahl.

- Die Methode der Marknagelung und der minimalen Invasivität kann eine ungünstige Reposition nicht kompensieren.

- Als Reosteosynthese kann die Kondylenplatte als Mittel der ersten Wahl empfohlen werden.

- Es sollten möglichst konsequent alle Optionen ausgenützt und eingesetzt werden: Dekortikation, ausreichend betonte Valgisation, Medialisierung des Schaftes, Zuggurtung und interfragmentäre Kompression.

\section{- Im vorliegenden Fall sind die Einzel- komponenten nur schrittweise und nicht konsequent genug eingesetzt worden. \\ - Letztendlich hat aber der zuletzt kon- sequente Einsatz all der beschriebe- nen Optionen zur kopferhaltenden Ausheilung geführt.}

\section{Prof. Dr. med. Dankward Höntzsch} Leitender Arzt

Abteilung für medizintechnische

Entwicklung

Berufsgenossenschaftliche Unfallklinik Tübingen

Schnarrenbergstraße 95

72076 Tübingen

E-Mail: dhoentzsch@bgu-tuebingen.de 